\title{
Análise de Custo-Minimização entre o Infliximabe (IFX) e o Adalimumabe (ADA) no Tratamento da Doença de Crohn (DC)
}

\author{
Cost-Minimization Analysis with Infliximab (IFX) and Adalimumab (ADA) \\ for the Treatment of Crohn's Disease (CD)
}

\author{
PAULO GUSTAVO KOTZE ${ }^{1}$; IDBLAN CARVALHO DE ALBUQUERQUE \\ ANTÔNIO CARLOS MORAES ${ }^{3}$; ANDREA VIEIRA ${ }^{4}$; FERNANDO DE SOUZA 5
}

\begin{abstract}
1. Chefe do Serviço de Coloproctologia do Hospital Universitário Cajuru (SeCoHUC) - PUCPR; Membro Titular da Sociedade Brasileira de Coloproctologia; ${ }^{2}$ Médico coloproctologista e responsável pelo Ambulatório de Doenças Inflamatórias Intestinais do Hospital Heliópolis - SP; Membro Titular da Sociedade Brasileira de Coloproctologia; ${ }^{3 .}$ Médico Gastroenterologista pela FBG; Membro do GEDIERJ; Diretor Clínico do Hospital Copa D'Or - RJ; 4. Médica assistente da Clínica de Gastroenterologia do Departamento de Medicina da Faculdade

de Ciências Médicas da Santa Casa de São Paulo - SP; Doutora pela FCMSCSP; 5. Professor Adjunto da

Disciplina de Clínica Cirúrgica - Serviço de Coloproctologia da UEM - PR; Membro Titular da Sociedade Brasileira de Coloproctologia.
\end{abstract}

KOTZE PG; ALBUQUERQUE IC; MORAES AC; VIEIRA A; SOUZA F. Análise de Custo-Minimização entre o Infliximabe (IFX) e o Adalimumabe (ADA) no Tratamento da Doença de Crohn (DC). Rev bras Coloproct, 2009;29(2): 158-168.

RESUMO: Introdução: há uma preocupação crescente com os custos da terapia biológica no tratamento da DC. O objetivo deste estudo foi simular o custo-minimização do tratamento contínuo com o IFX e com o ADA em portadores de DC, num período de 1 ano, em variadas faixas de peso. Método: estudo farmacoeconômico de custo-minimização na simulação de tratamento com agentes biológicos de pacientes portadores de DC, com pesos diferentes. Os custos foram baseados no preço dos dois medicamentos isoladamente (IFX E ADA). Resultados: o custo do tratamento com IFX (sistema público) foi de R\$29.411,12 (entre 20 e $40 \mathrm{~kg}$ ), R\$44.116,68 (entre 41 e $60 \mathrm{~kg}$ ), R\$58.822,24 (entre 61 e $80 \mathrm{~kg}$ ) e R\$ 73.527,80 (entre 81 e $100 \mathrm{~kg}$ ). O custo com ADA foi de $\mathbf{R} \$ \mathbf{5 2 . 0 4 5 , 1 6}$, independentemente do peso. A análise do sistema privado e situações de perda de resposta encontram-se descritas no artigo. Conclusões: houve menores custos com o uso do IFX abaixo de $60 \mathrm{~kg}$, e com o ADA acima deste peso. Em simulação de perda de resposta ao IFX, houve menores custos absolutos com a troca para ADA do que aumento de dose do IFX, entre 40 e $100 \mathrm{~kg}$.

Descritores: Doença de Crohn; Terapia Biológica; Fator de Necrose Tumoral Alfa; Economia; Custos e Análise de Custo.

\section{INTRODUÇÃO}

A doença de Crohn (DC) é uma doença inflamatória transmural e recidivante do trato gastrointestinal, que pode acometer qualquer segmento do tubo digestório, da boca ao ânus ${ }^{1,2}$. É ca- racterizada por inflamação descontínua dos segmentos acometidos, com formas distintas de manifestações em cada indivíduo (luminal, penetrante ou fistulizante) $)^{1,3}$.

Desde a sua descrição inicial em 1932 por Burrill B. Crohn et al. ${ }^{4}$, esta doença tem se compor-

Trabalho realizado no Serviço de Coloproctologia do Hospital Universitário Cajuru (SeCoHUC) - PUCPR - Curitiba - PR - Brasil.

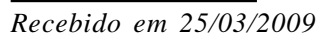

Aceito para publicação em 28/04/2009 
tado como um enigma para gastroenterologistas e imunologistas $^{5}$. Com o desenvolvimento de modernas e minuciosas técnicas laboratoriais, associadas ao avanço da pesquisa clínica, novos caminhos foram percorridos no conhecimento de sua etiopatogenia. Como consequência, surgiram novas terapias com bons resultados no controle da atividade da doença ${ }^{6}$.

O fator de necrose tumoral alfa (TNF- $\alpha)$ é uma citocina fundamental na origem, amplificação e manutenção da disfunção inflamatória observada nos portadores de $\mathrm{DC}^{7}$. A terapia biológica com medicamentos anti-TNF- $\alpha$ tem demonstrado comprovada eficácia clínica na indução e manutenção da remissão da doença ${ }^{3}$. O infliximabe (IFX) é um anticorpo monoclonal quimérico com $25 \%$ da sua estrutura formada por proteína murínica e $75 \%$ por proteína humana, de uso endovenoso ${ }^{8}$. Sua utilização para o tratamento da DC foi aprovada em 1998 nos Estados Unidos, e atualmente, milhares de pacientes já foram tratados com este medicamento. A experiência clínica com dez anos de uso do IFX é ampla, e há bom conhecimento de dados de sua eficácia e de sua segurança na prática clínica9 ${ }^{9}$ O adalimumabe (ADA) é um anticorpo monoclonal $100 \%$ humano, de uso subcutâneo, liberado nos Estados Unidos para o tratamento da DC em $2007^{10}$, que igualmente apresenta importantes dados de eficácia e segurança já publicados no tratamento destes pacientes ${ }^{11}$.

Há extensa experiência com estas duas drogas pelo seu uso mais antigo na reumatologia, no tratamento da artrite reumatoide, psoriática e na espondilite anquilosante. Ambas podem ser utilizadas para o tratamento da Doença de Crohn de moderada a grave, na forma luminal ou fistulizante, com eficácia clínica comprovada $^{10,12-14}$. O ADA apresentou ainda, significativa resposta em pacientes que não mais responderam a infusões do IFX, em relação ao placebo ${ }^{15}$.

Mesmo sendo medicamentos de alto custo no tratamento da DC, os anticorpos monoclonais representam um grande avanço na terapêutica, pois há significativa diminuição no número de internações, cirurgias e exames complementares no seguimento destes pacientes ${ }^{16,17}$. Além disso, apresentam maior eficácia em relação à terapia convencional ${ }^{12,14}$. No Brasil, o uso do IFX e do ADA para o tratamento da DC está regulamentado pela Agência Nacional de Vigilância Sanitária (ANVISA) desde 2000 e 2007, respectivamente.
Atualmente há preocupação crescente com os custos de determinados tratamentos, e a cada ano, novos estudos de farmacoeconomia vêm sendo publicados para que se defina o real benefício econômico de cada medicamento. Não se trata de simples comparações entre preços, mas sim, de análises multifatoriais complexas, baseadas em modelos econômicos, que tentam chegar a um valor real para o custo de um tratamento por determinado período de tempo. Dentre as variadas comparações de farmacoeconomia, a análise de custo-minimização é uma das mais simplificadas, e baseia-se no pressuposto de que as duas drogas comparadas tenham a mesma eficácia clínica ${ }^{18}$. Não se avalia resposta, efeitos adversos, custos de infusões, entre outros. Analisa-se somente os custos das unidades dos medicamentos utilizados, isoladamente. Um estudo comparativo de custo-minimização entre o IFX, ADA e etanercept foi publicado na análise do tratamento da artrite reumatoide (AR), em nosso meio, com diferentes dosagens e esquemas terapêuticos ${ }^{18}$. Não há estudos nacionais que comparem os custos do IFX e do ADA no tratamento da DC.

Como o IFX é um fármaco com dosagem pesodependente ( 1 frasco para cada $20 \mathrm{~kg}$ de peso), passa a ser de interesse fundamental em uma análise farmacoeconômica qual seria o peso médio de um paciente portador de DC submetido à terapia biológica. Esta faixa média de peso varia entre 69 e $75 \mathrm{~kg}$, conforme os estudos controlados consultados na literatura internacional. Isto faz desta faixa de peso um dado interessante na análise proposta por esta simulação. Os dados dos principais estudos que relatam o peso médio em suas análises estão ilustrados na tabela 1 , com seus respectivos autores.

O objetivo principal deste estudo foi simular o custo-minimização do tratamento contínuo em portadores de DC com o IFX e com o ADA, num período de 1 ano em variadas faixas de peso, na indução da remissão e na manutenção da mesma.

Os objetivos secundários foram:

a) avaliar situações de perda de resposta às duas drogas (interrupções do tratamento), baseadas nos dados dos principais estudos das mesmas, com análise dos custos das terapias de resgate propostas.

b) avaliar todos os resultados encontrados especificamente na faixa de peso entre 61 e $80 \mathrm{~kg}$ (faixa de peso médio encontrada na maioria dos estudos sobre terapia biológica na DC na literatura internacional). 
Tabela 1 - Peso médio dos pacientes com DC submetidos à terapia biológica. Principais estudos da literatura internacional. NATA=Natalizumabe.

\begin{tabular}{lllcc}
\hline Estudo & Autor & Ano & Peso médio (kg) & Droga \\
\hline TARGAN (cA2) & Targan et al. $^{8}$ & 1997 & 70,52 & IFX \\
ACCENT I & Hanauer et al. ${ }^{12}$ & 2002 & 71,1 & IFX \\
ACCENT II & Sands et al. ${ }^{13}$ & 2004 & 73,4 & IFX \\
CLASSIC I & Hanauer et al. ${ }^{10}$ & 2006 & 75,25 & ADA \\
CLASSIC II & Sandborn et al. $^{19}$ & 2006 & 72 & ADA \\
CHARM & Colombel et al. $^{14}$ & 2007 & 69,97 & ADA \\
GAIN & Sandborn et al. & & \\
ENACT 1 E 2 & Sandborn et al. & & 72 & ADA \\
\hline
\end{tabular}

\section{MÉTODO}

Trata-se de um estudo farmacoeconômico descritivo de uma simulação de tratamento de pacientes portadores de DC, com pesos diferentes. Os casos simulados foram determinados como candidatos à terapia biológica com as duas drogas liberadas pela ANVISA no Brasil: Infliximabe (Remicade ${ }^{\circledR}$ Mantecorp do Brasil) e Adalimumabe (Humira® Abbott Laboratórios do Brasil). Os custos analisados foram apenas das drogas biológicas isoladas, não sendo somados os custos indiretos e custos com imunossupressores, infusões ou outras drogas utilizadas conjuntamente para o tratamento da DC. A análise farmacoeconômica proposta foi a de custominimização.

Foram pré-determinadas situações de pacientes portadores de DC com variadas faixas de peso, e simulados tratamentos de indução da remissão com manutenção por 56 semanas, sem interrupções, de acordo com a literatura ${ }^{12,14}$. Os intervalos de peso utilizados nesta simulação foram entre 20 e $40 \mathrm{~kg}$, entre 41 e $60 \mathrm{~kg}$, entre 61 e $80 \mathrm{~kg}$ e entre 81 e $100 \mathrm{~kg}$, totalizando quatro grupos. Esta divisão por faixa de peso é justificada pelas propriedades farmacológicas do IFX, com dosagem peso-dependente. Sua apresentação exige que cada frasco da medicação seja utilizado para cada $20 \mathrm{~kg}$ de peso $(5 \mathrm{mg} / \mathrm{kg}$ de peso - frascos de 100 $\mathrm{mg}$ ).

As doses de indução da remissão do IFX foram simuladas de acordo com o estudo ACCENT I (5 $\mathrm{mg} / \mathrm{kg}$ de peso EV nas semanas 0,2 e 6$)^{12}$. Já as doses de indução da remissão com o ADA foram simuladas de acordo com o estudo CLASSIC I (160 mg SC na semana 0 e $80 \mathrm{mg}$ na semana 2$)^{10}$. As doses de manutenção por 1 ano para o IFX foram simuladas de acordo com os achados do estudo ACCENT I $(5 \mathrm{mg} / \mathrm{kg}$ de peso EV a cada 8 semanas) ${ }^{12}$. Para o ADA, esta dosagem foi baseada nos dados do estudo CHARM, com utilização de $40 \mathrm{mg} \mathrm{SC}$ a cada duas semanas pelo período de 56 semanas $^{14}$ (tabela 2).

$\mathrm{O}$ custo das medicações foi baseado na revista Kairos, do mês de fevereiro de $2009^{21}$, e foi diferente nos dois sistemas de saúde simulados. No sistema público de saúde, utilizou-se o preço de fábrica das medicações (PF), com isenção de 18\% de ICMS (existente em muitos estados brasileiros) e de $24,92 \%$ de CAP (Coeficiente de Adequação de Preço - portaria do ministério da saúde para medicamentos de alto custo) ${ }^{22}$. No sistema privado (convênios e planos de saúde), a análise foi baseada no preço máximo ao consumidor (PMC).

$\mathrm{Na}$ análise do sistema público, o custo de cada frasco de IFX (Remicade ${ }^{\circledR}$ - Mantecorp do Brasil) foi

Tabela 2 - Esquemas de indução da remissão e manutenção de remissão das duas drogas.

\begin{tabular}{lcc}
\hline & \multicolumn{1}{c}{ Indução da remissão } & Manutenção (1 ano) \\
\hline Infliximabe & $5 \mathrm{mg} / \mathrm{kg}$ de peso nas semanas 0, 2 e 6 & $5 \mathrm{mg} / \mathrm{kg}$ de peso a cada 8 semanas \\
Adalimumabe & $160 \mathrm{mg}$ na semana 0 e 80 mg na semana 2 & $40 \mathrm{mg}$ a cada 2 semanas \\
\hline
\end{tabular}


de $\mathrm{R} \$ 1.633,95$ (PF com isenção de ICMS e redução de $24,92 \%$ referente ao CAP) e o preço da seringa pré-preenchida de ADA (Humira ${ }^{\circledR}$ - Abbott Laboratórios do Brasil) foi da mesma maneira definido em $\mathrm{R} \$$ 1.577,13. Na análise do sistema privado (PMC), o custo de cada frasco de IFX foi de R $\$ 3.668,79$ e o preço da seringa pré-preenchida de ADA foi de $\mathrm{R} \$ 3.541,20$ (tabela 3).

O cálculo do custo total da indução da remissão, e da manutenção por 56 semanas foi realizado para as duas drogas separadamente, para posterior comparação nas faixas de peso determinadas.

Foram simuladas ainda, situações de perda de resposta às duas drogas, visto tratar-se de situação que pode ocorrer na prática clínica diária, com interrupção do tratamento e necessidade de terapias de resgate. Para esta análise, utilizou-se dados do estudo o ACCENT I para o IFX, com pressuposto da perda de resposta na semana $22^{12}$. Para o ADA, utilizou-se o mesmo período na simulação, por não haver na literatura dados claros sobre o índice da perda de resposta a esta droga. Não se considerou a porcentagem da perda de resposta ou intolerância às duas drogas, visto tratar-se de análise farmacoeconômica de custo-minimização, onde a eficácia não é incluída na análise. Apenas o período médio da interrupção dos tratamentos foi considerado (22 semanas).

No caso da perda de resposta ou intolerância ao IFX, há 3 possibilidades de tratamento de resgate: aumento de dose para $10 \mathrm{mg} / \mathrm{kg}$ de peso a cada $8 \mathrm{se}-$ manas ${ }^{23}$; manutenção da dose de $5 \mathrm{mg} / \mathrm{kg}$ de peso com redução do intervalo para 6/6 semanas ${ }^{23}$; e troca para ADA $40 \mathrm{mg}$ a cada 2 semanas ${ }^{15}$. No caso da perda de resposta ao ADA, a proposta de terapia de resgate simulada foi a redução do intervalo para $40 \mathrm{mg}$ por semana ${ }^{14}$. Estas alternativas, com o número de unidades utilizadas em cada possibilidade de resgate de tratamento, estão ilustradas na tabela 5, em conjunto com os resultados.

\section{RESULTADOS}

Os números de unidades de cada medicamento utilizados no período de 56 semanas, com os respectivos custos dos tratamentos, nos sistemas público e privado, de acordo com a metodologia de cálculo utilizada previamente descrita, estão ilustrados na tabela 4. Num período de 56 semanas, somadas as dosagens de indução da remissão com a manutenção programada, são utilizadas 33 seringas pré-preenchidas de ADA, independentemente da faixa de peso. Neste mesmo período, são utilizados 18 frascos de IFX entre 20 e 40 $\mathrm{kg} ; 27$ frascos entre 41 e $60 \mathrm{~kg} ; 36$ frascos entre $61 \mathrm{e}$ $80 \mathrm{~kg}$ e 45 frascos entre 81 e $100 \mathrm{~kg}$.

Os achados da simulação de perda de resposta estão detalhados na tabela 5 , nas diversas faixas de peso simuladas, com a descrição dos dois sistemas estudados (público e privado) e com os números de cada unidade de medicamentos utilizados (IFX e ADA).

As figuras 1 e 2 ilustram os achados da simulação dos custos de 56 semanas com IFX nas quatro faixas de peso, e os custos com o tratamento com ADA, nos sistemas público e privado, respectivamente. Observa-se um maior custo do tratamento com IFX a partir dos $61 \mathrm{~kg}$ de peso, comparado ao custo do ADA. Notase que na faixa de peso entre 61 e $80 \mathrm{~kg}$ (figura 3), a mais comumente descrita nos estudos da literatura para

Tabela 3 - Cálculos e valores de cada unidade de medicamento (Frascos de IFX; seringas pré-preenchidas de ADA). PF: Preço de fábrica; ICMS: imposto sobre circulação de mercadorias e serviços; CAP: coeficiente de adequação do preço; PMC: preço máximo ao consumidor.

\begin{tabular}{llcc}
\hline Medicamento & Sistema & Cálculo & Valor por unidade \\
\hline INFLIXIMABE (IFX) $100 \mathrm{mg} / \mathrm{ml}$ & Público & PF - ICMS (18\%) - & $\mathrm{R} \$ 1.633,95$ \\
& Privado & CAP $(24,92 \%)$ & $\mathrm{R} \$ 3.668,79$ \\
ADALIMUMABE (ADA) 40mg & Público & PF - ICMS (18\%) - & $\mathrm{R} \$ 1.577,13$ \\
& & CAP $(24,92 \%)$ & $\mathrm{R} \$ 3.541,20$ \\
\hline
\end{tabular}


pacientes com terapia biológica em DC, os custos com o IFX são superiores aos custos do tratamento com ADA, com uma diferença de $13 \%$. Esta diferença é ainda maior na faixa de peso entre 81 e $100 \mathrm{~kg}$, na faixa de $41 \%$.

Nas diversas situações de perda de resposta simuladas, as figuras de 4 a 7 ilustram os valores de custos encontrados separadamente. Os 3 pares de colunas da esquerda representam a perda de resposta ao IFX, com as respectivas estratégias de resgate de tratamento (aumento de dose de IFX para $10 \mathrm{mg} / \mathrm{Kg}$, redução do intervalo de administração de IFX para 6/6 semanas e troca de IFX para ADA 40 mg a cada 2 semanas, respectivamente). O quarto par de colunas representa uma perda de resposta ao ADA a partir da semana 22 do tratamento, com o resgate através da administração de $40 \mathrm{mg}$ semanalmente, conforme a metodologia adotada (sem uso do IFX). São demonstrados os achados da simulação de custo-minimização nos sistemas público (legenda azul), e privado (legenda amarela), nos mesmos gráficos.

Conforme observado, nestas situações de interrupção dos tratamentos e necessidade de terapias de resgate para manutenção da remissão os achados são diferentes nas variadas faixas de peso. No aumento de dose de IFX para $10 \mathrm{mg} / \mathrm{kg}^{23}$, estes valores mostram importantes conclusões. Entre 20 e $40 \mathrm{~kg}$,

Tabela 4 - Número de unidades de cada medicamento utilizadas no período de um ano (indução da remissão e manutenção). Custos em reais $(R \$)$.

\begin{tabular}{lccc}
\hline Droga / faixa de peso & $\begin{array}{c}\text { Número de unidades } \\
\text { administradas }\end{array}$ & $\begin{array}{c}\text { Custo total do } \\
\text { tratamento (sistema público) }\end{array}$ & $\begin{array}{c}\text { Custo total do } \\
\text { tratamento (sistema privado) }\end{array}$ \\
\hline IFX/ entre 20 e 40 kg & 18 & $29.411,12$ & $66.038,22$ \\
IFX/ entre 41 e $60 \mathrm{~kg}$ & 27 & $44.116,68$ & $99.057,33$ \\
IFX/ entre 61 e $80 \mathrm{~kg}$ & 36 & $58.822,24$ & $132.076,44$ \\
IFX/ entre 81 e $100 \mathrm{~kg}$ & 45 & $73.527,80$ & $165.095,55$ \\
ADA/ qualquer faixa de peso & 33 & $52.045,16$ & $116.859,44$ \\
\hline
\end{tabular}

Tabela 5 - Esquemas de tratamento de resgate em caso de perda de resposta ou intolerância aos medicamentos, a partir da semana 22. Custos em reais $(R \$)$.

\begin{tabular}{|c|c|c|c|c|c|c|}
\hline $\begin{array}{l}\text { Perdade } \\
\text { resposta } \\
\text { (medicamento) }\end{array}$ & $\begin{array}{c}\text { Opçãode terapia } \\
\text { de resgate após } \\
\text { a semana } 22\end{array}$ & $\begin{array}{c}\text { Faixas } \\
\text { de peso } \\
\text { estudadas }\end{array}$ & $\begin{array}{c}\text { Númerode } \\
\text { unidades } \\
\text { deIFX }\end{array}$ & $\begin{array}{c}\text { Númerode } \\
\text { unidades } \\
\text { deADA }\end{array}$ & $\begin{array}{c}\text { Custo total } \\
\text { dotratamento } \\
\text { (sistema público) }\end{array}$ & $\begin{array}{c}\text { Custo total } \\
\text { dotratamento } \\
\text { (sistema privado) }\end{array}$ \\
\hline \multirow[t]{12}{*}{ IFX } & IFX $10 \mathrm{mg} / \mathrm{kg}$ & Entre $20 \mathrm{e} 40 \mathrm{~kg}$ & 28 & - & $45.750,63$ & $102.726,12$ \\
\hline & $8 / 8 \mathrm{sem}$ & Entre 41 e $60 \mathrm{~kg}$ & 42 & & $68.625,94$ & $154.089,18$ \\
\hline & & Entre 61 e $80 \mathrm{~kg}$ & 56 & & $91.501,26$ & $205.452,24$ \\
\hline & & Entre 81 e $100 \mathrm{~kg}$ & 70 & & $114.376,57$ & $256.815,30$ \\
\hline & IFX 5 mg/kg & Entre 20 e $40 \mathrm{~kg}$ & 20 & - & $32.679,02$ & $73.375,80$ \\
\hline & $6 / 6 \mathrm{sem}$ & Entre 41 e $60 \mathrm{~kg}$ & 30 & & $44.116,68$ & $99.057,33$ \\
\hline & & Entre 61 e $80 \mathrm{~kg}$ & 40 & & $65.358,04$ & $146.751,60$ \\
\hline & & Entre 81 e $100 \mathrm{~kg}$ & 50 & & $81.697,55$ & $183.439,50$ \\
\hline & ADA $40 \mathrm{mg}$ & Entre 20 e $40 \mathrm{~kg}$ & 8 & 14 & $47.768,43$ & $107.256,69$ \\
\hline & $2 / 2 \mathrm{sem}$ & Entre 41 e $60 \mathrm{~kg}$ & 12 & & $54.304,23$ & $121.931,85$ \\
\hline & & Entre 61 e $80 \mathrm{~kg}$ & 16 & & $60.840,03$ & $136.607,01$ \\
\hline & & Entre 81 e $100 \mathrm{~kg}$ & 20 & & $67.375,87$ & $151.282,17$ \\
\hline $\mathrm{ADA}$ & $\mathrm{ADA} 40 \mathrm{mg} / \mathrm{sem}$ & - & - & 50 & $78.856,50$ & $177.060,00$ \\
\hline
\end{tabular}


com perda de resposta ao IFX, há menores custos com o ajuste de dose do IFX para $10 \mathrm{mg} / \mathrm{kg}$ do que com a troca para ADA. Entretanto, esta diferença é pequena $(4,4 \%$ mais alto para o $\mathrm{ADA})$. A partir da faixa de peso acima dos $41 \mathrm{~kg}$, há menores custos com a troca de anti-TNF para ADA do que com o dobro da dose do IFX. Este custo mais alto para o

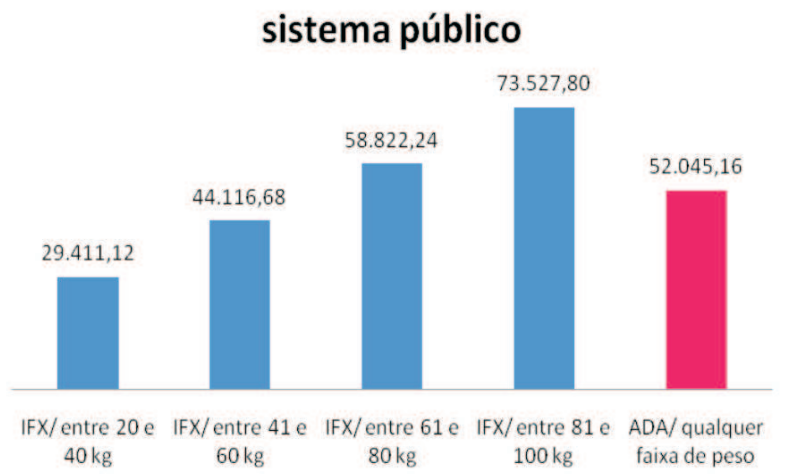

Figura 1 - Custos anuais de tratamento - sistema público.

\section{sistema privado}

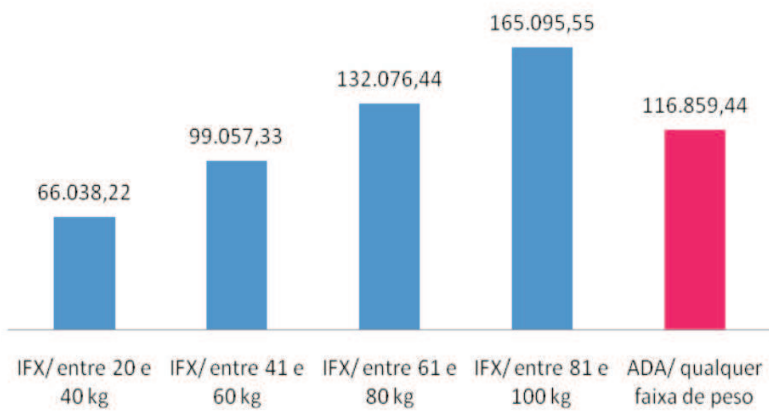

Figura 2 - Custos anuais de tratamento - sistema privado.

\section{Peso entre 61 e 80 kg}

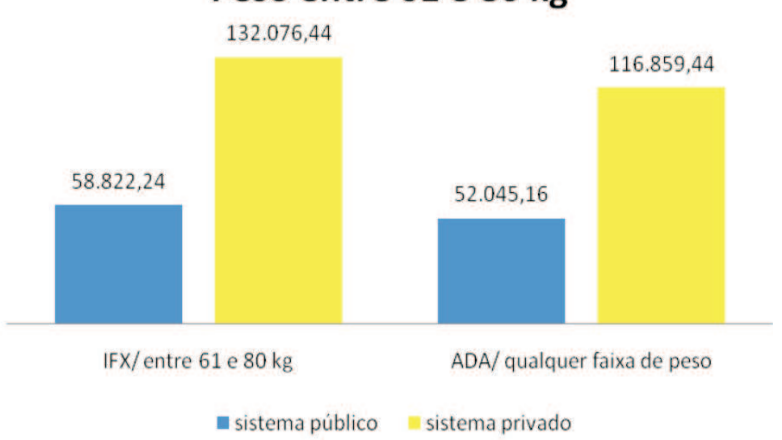

Figura 3 - Faixa de peso entre 61 e $80 \mathrm{~kg}$ (peso médio de pacientes com DC e terapia biológica na literatura internacional) - achados específicos (sistemas público e privado). Diferença de 11,92\%, com menores custos com o ADA.

\section{Entre 20 e $40 \mathrm{~kg}$}

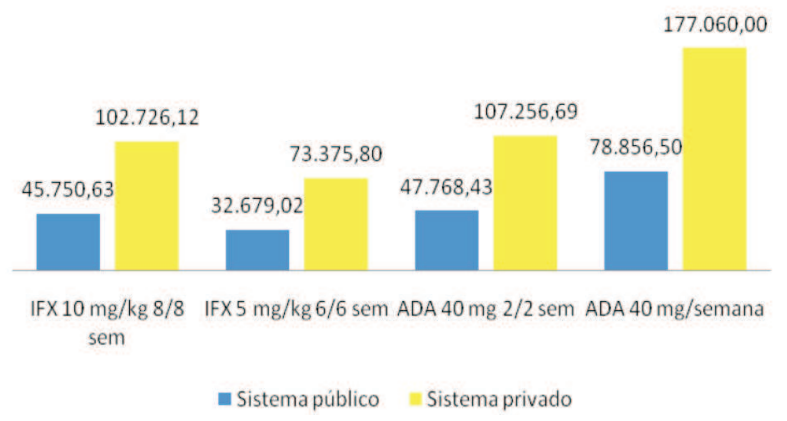

Figura 4 - Perda de resposta entre 20 e $40 \mathrm{~kg}$. Custos em reais $(R \$)$.

\section{Entre 41 e $60 \mathrm{~kg}$}

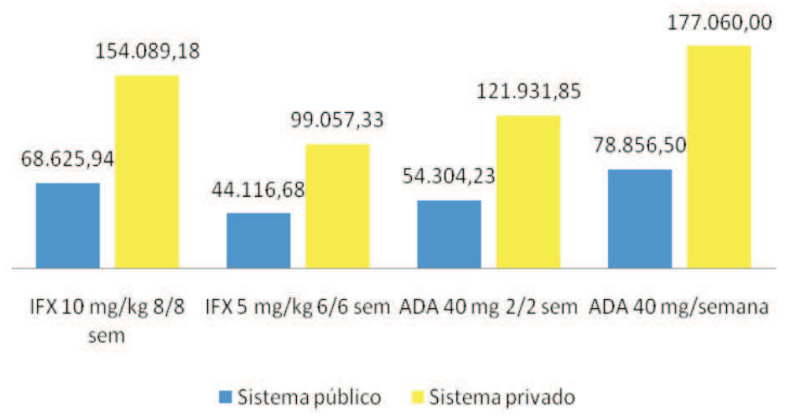

Figura 5 - Perda de resposta entre 41 e $60 \mathrm{~kg}$. Custos em reais $(R \$)$.

\section{Entre 61 e $80 \mathrm{~kg}$}

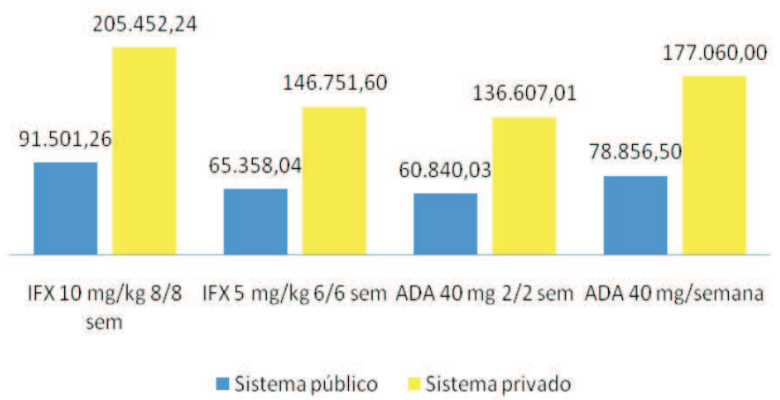

Figura 6 - Perda de resposta entre 61 e $80 \mathrm{~kg}$. Custos em reais $(R \$)$.

\section{Entre 81 e 100 kg}

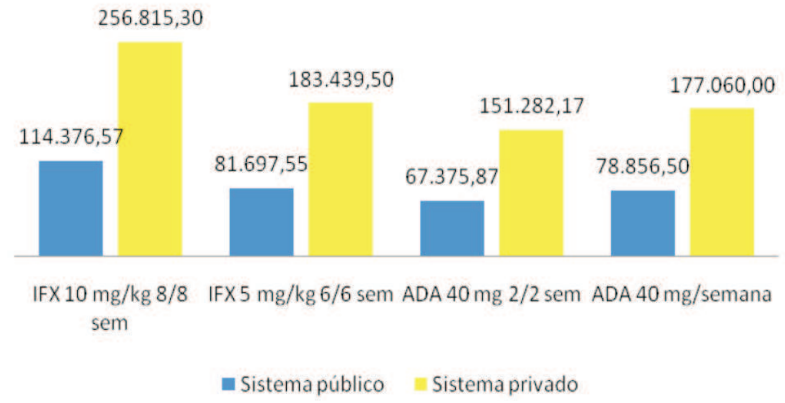

Figura 7 - Perda de resposta entre 81 e $100 \mathrm{~kg}$. Custos em reais $(R \$)$. 
IFX cresce de acordo com a faixa de peso estudada (26,4\% entre 41 e $60 \mathrm{~kg} ; 50,4 \%$ entre 61 e $80 \mathrm{~kg}$ e $69,7 \%$ entre 81 e $100 \mathrm{~kg}$ ).

Se a estratégia de redução do intervalo das aplicações do IFX for adotada, com doses a cada 6 semanas mantendo-se $5 \mathrm{mg} / \mathrm{kg}$ de peso ${ }^{23}$, há menores custos com a troca de anti-TNF para ADA somente a partir dos $61 \mathrm{~kg}$. A diferença de custos entre estas estratégias é de $7 \%$ entre 61 e $80 \mathrm{~kg}$ e de $21,2 \%$ entre 81 e $100 \mathrm{~kg}$, mais alta para o IFX.

Caso a perda de resposta ocorra com o uso do ADA, com a estratégia de dobrar sua dose para 40 $\mathrm{mg} / \mathrm{semana}^{14}$ a partir da semana 22 de tratamento, os custos desta terapia de resgate aumentam consideravelmente. Porém, comparando-se dobrar as doses do IFX com as do ADA entre si, observa-se que os custos são menores no tratamento com ADA a partir dos 61 $\mathrm{kg}$ de peso. A diferença percentual entre iniciar um tratamento e dobrar sua dose a partir da semana 22 é de $16 \%$ entre 61 e $80 \mathrm{~kg}$ e de $45 \%$ entre 81 e $100 \mathrm{~kg}$, mais alto com IFX. Portanto, a partir dos $61 \mathrm{~kg}$ de peso, os custos são menores no tratamento com ADA se comparados ao IFX, quando não se pretende trocar de agente anti-TNF na terapia.

Nas faixas de peso abaixo de $40 \mathrm{~kg}$, observa-se que os custos com o IFX serão sempre menores, nas duas terapias de resgate, se comparados à troca de anti-TNF para o ADA. Portanto, esta análise de custo-minimização demontra vantagens nítidas de redução de custos para o tratamento com o IFX na faixa etária pediátrica e em pacientes com desnutrição grave, por exemplo. As doses do uso do ADA em pediatria ainda não são definidas por estudos clínicos controlados, apenas algumas séries de casos foram publicadas com utilização da dose normal de adultos ${ }^{24}$.

\section{DISCUSSÃO}

Os médicos que tratam a doença de Crohn, atualmente, têm de se concentrar nas variadas formas clínicas da doença, dificuldades de diagnóstico e na ampla gama de tratamentos disponíveis. Trata-se de uma afecção de difícil manuseio, o que é feito por poucos profissionais que realmente se interessam por este grande desafio. Todavia, poucas são as preocupações com os custos do tratamento destes pacientes, visto que há escassez na literatura nacional de publicações a este respeito.
A terapia biológica é a forma mais moderna de tratamento da DC, e é acompanhada de custos elevados se comparada ao tratamento convencional, com uso de corticoides, antibióticos, derivados do 5-ASA e imunossupressores. Esta terapia apresenta resultados excelentes nas formas moderadas a graves da doença, e é arma importante no arsenal terapêutico do combate à inflamação típica desta enfermidade 6 . O uso dos agentes biológicos vem aumentando na prática clínica diária, e estudos relatam algumas vantagens até na utilização destas drogas em períodos precoces da doença, em subgrupos determinados de pacientes ${ }^{25}$. Portanto, não se pode atualmente dissociar o tema de doenças inflamatórias intestinais da terapia biológica, foco das principais pesquisas terapêuticas na área nos últimos anos.

Quanto custa um tratamento com a terapia biológica no Brasil? Esta pergunta era respondida, há alguns anos, com uma simples resposta: muito caro. Entretanto, estudos de farmacoeconomia, inicialmente com o IFX, posteriormente com o ADA, vieram a mostrar outra realidade. Sabe-se que há redução do número de internamentos e de cirurgias com o uso dos biológicos, se comparados à terapia convencional ${ }^{16,17}$. Há consequente redução do número de exames complementares solicitados, assim como dos gastos hospitalares. Atualmente, por esta razão, o cenário acena para uma mudança na maneira de se encarar os custos de um tratamento. Os biológicos são medicações mais caras do que as drogas da terapia convencional. Porém, seu uso diminui os custos globais no tratamento contínuo da doença, por manter o paciente em remissão por mais tempo, impedindo crises de agudização e suas consequências.

Esta análise teve como proposta comparar os custos das duas drogas biológicas disponíveis no Brasil para o tratamento da DC. Para este fim, a análise de custo-minimização foi utilizada por ser a mais simples, e foram adotados somente os custos das drogas isoladamente, sem levar em consideração outros gastos (infusão, internação, materiais e medicamentos associados) ou custos indiretos, como afastamento do trabalho. Independentemente do sistema de saúde simulado, as diferenças percentuais de custos entre as drogas se mantêm.

Os achados desta simulação acenam para um menor custo da terapia biológica para a DC com o ADA do que com o IFX, em pacientes adultos, com peso 
médio acima de $60 \mathrm{~kg}$. Entretanto, se os custos indiretos fossem somados, esta diferença de $13 \%$ poderia ser até mais acentuada. A necessidade da infusão endovenosa do IFX, com seus custos de monitoração, horas trabalhadas de profissionais da área de saúde, tempo decorrente para a administração do medicamento e afastamento do trabalho, são fatores que poderiam aumentar ainda mais esta diferença de valores. O ADA é administrado pelo próprio paciente, sem necessidade de infusão em unidade ambulatorial ou hospitalar (medicação subcutânea), tendo com isso, menores custos associados.

Por outro lado, nas menores faixas de peso, os custos são claramente mais reduzidos com o uso do IFX do que as administrações subcutâneas do ADA. Abaixo dos $60 \mathrm{~kg}$, esta diferença é clara. Isto se deve ao fato da dose do IFX ser peso-dependente, conforme previamente citado. Para pacientes pediátricos, por exemplo, os custos com o IFX são significativamente menores, além de ser a única droga biológica aprovada pela ANVISA para este fim. A dose de ADA para pacientes pediátricos não está ainda definida em estudos controlados.

Salienta-se que pacientes com peso entre 41 e $60 \mathrm{~kg}$, que utilizariam três frascos de IFX, podem ter seu peso recuperado e aumentado durante o tratamento de um ano de duração. Isto se deve a uma esperada melhora no estado nutricional que comumente acompanha pacientes com resposta ou remissão durante o tratamento. Este aumento de peso, se ultrapassados os $60 \mathrm{~kg}$, levaria a uma necessidade de aumento do número de frascos de IFX, e este ajuste de dose pode ocorrer durante o ano do uso da medicação. Este fator obviamente aumentaria os custos com o uso do IFX, e não foi computado nesta análise.

Outro ponto que deve ficar claro é que este tipo de análise, de custo-minimização, não imputa a taxa de resposta dos pacientes aos medicamentos. Sabe-se que as taxas de resposta e remissão ao IFX e ADA são semelhantes, com variadas metodologias $\operatorname{adotadas}^{8-17}$. Entretanto, pequena divergência de números pode ser encontrada ao se analisar minuciosamente os estudos controlados. Uma análise que colocasse em vigência a resposta dos pacientes seria uma análise de custo-efetividade, outra possibilidade de modelo farmacoeconômico. Entretanto, este foco foge aos objetivos traçados para o presente estudo.
Um estudo nacional analisou o custominimização entre os tratamentos biológicos para pacientes portadores de artrite reumatoide $(\mathrm{AR})^{18}$. As drogas analisadas neste trabalho foram o IFX, o ADA e o etanercept. A conclusão deste estudo foi que o IFX apresentava menores custos em relação às outras duas drogas, num peso médio considerado de $65 \mathrm{~kg}$. Um simples fator pode justificar estes achados. A dose de IFX preconizada para AR é a de 3 $\mathrm{mg} / \mathrm{kg}$ de peso, o que reduz o número de frascos por infusão, enquanto que a dose de ADA é $40 \mathrm{mg}$ a cada 2 semanas. Os achados da presente análise, no tratamento da DC, vão contra os encontrados na simulação de $\mathrm{AR}$, principalmente pela maior dosagem requerida no tratamento da DC $(5 \mathrm{mg} / \mathrm{kg}$ de peso). Houve nítida redução de custos com o ADA na faixa de peso médio de DC, entre 61 e $80 \mathrm{~kg}$. Este estudo, publicado em 2005, talvez explique o cenário atual de dificuldades na liberação do ADA nos sistemas público e privado de saúde no Brasil, para o tratamento da DC. Têm-se a idéia que o tratamento com ADA seria mais oneroso, pela experiência prévia com a AR. Entretanto, os achados da presente análise farmacoeconômica mostram um cenário oposto, no que tange a DC.

Em relação à interrupção de um tratamento com drogas anti-TNF, ela pode ocorrer por duas causas: efeitos adversos do tratamento (reações infusionais, alérgicas, infecções oportunistas, entre outros) ou perda de resposta (piora do quadro clínico independentemente da continuidade do tratamento). Discute-se atualmente na literatura, qual a melhor estratégia para a terapia de resgate destes pacientes ${ }^{28}$. Há evidências que o aumento de dose do IFX para 10 $\mathrm{mg} / \mathrm{kg}$, ou redução do seu intervalo para $6 / 6$ semanas pode causar nova remissão e melhora, nos casos de perda de resposta à droga. Há também dados que comprovam melhora clínica e remissão com a troca do IFX para o $\mathrm{ADA}^{15}$. Entretanto, o que seria melhor? O que seria menos oneroso para os sistemas de saúde?

Sugere-se que a troca de medicação possa trazer menor resposta, e que os pacientes previamente expostos a um agente anti-TNF têm menores índices de remissão do que os nunca antes expostos a estas drogas ${ }^{15,25}$. Entretanto, não há estudos controlados na literatura que tenham comparado diretamente as opções terapêuticas para a perda de resposta ao IFX (aumento de dose versus 
troca de agente anti-TNF), em termos de remissão ou resposta.

Todavia, os achados da presente análise mostram que os custos com a troca de IFX para ADA são menores, com uma perda de resposta simulada na semana 22. Isto fica evidente já a partir dos $40 \mathrm{~kg}$ de peso, com diferenças de custos ainda maiores nos pacientes mais pesados. A troca de anti-TNF passa a ser uma opção menos onerosa, com menores gastos para os sistemas de gestão de saúde.

Analisando-se a perda de resposta às duas drogas (IFX e ADA), com a suposição de que não se opte por trocar de medicação, o aumento da dose do IFX para $10 \mathrm{mg} / \mathrm{kg}$ fica mais oneroso que a diminuição do intervalo da dose do ADA para $40 \mathrm{mg}$ por semana, a partir dos $60 \mathrm{~kg}$ de peso. Este ponto precisa ficar destacado entre os resultados desta análise, pois no peso médio dos pacientes com DC (entre 61 e $80 \mathrm{~kg}$ ), é menos oneroso se iniciar uma terapia com ADA do que com IFX, sem incluir nesta análise dados de resposta clínica ou comodidade de administração de medicamentos. Sabe-se que a perda de resposta ao IFX pode atingir índices alarmantes, de até $77 \%$ em dois anos ${ }^{27}$. Os dados de perda de resposta ao ADA ainda não são claros na literatura. Têm-se somente as evidências de que $27 \%$ dos pacientes do estudo CHARM passaram para o braço aberto e redução do intervalo de dose para $40 \mathrm{mg}$ por semana ${ }^{14}$. Estas taxas de perda de resposta não foram imputadas na presente análise de custo-minimização, entretanto, podem ter influência importante nos gastos por respondedor ou por paciente em remissão ao final de um ano de tratamento.

Há um estudo americano de custoefetividade que compara custos, no caso de perda de resposta ao IFX, com troca para ADA ou com aumento da dose de IFX para $10 \mathrm{mg} / \mathrm{kg}^{28}$, levandose em consideração o desfecho econômico (avaliação do tipo QALY - quality adjusted life-year). Em custos absolutos por pacientes tratados, houve uma diferença de $36,2 \%$ de redução de custos com a troca para o ADA, o que corrobora os achados da presente simulação. Entretanto, salienta-se que este estudo americano é uma análise que leva em conta fatores de resposta ao tratamento. Apesar do maior custo, o complexo modelo utilizado sugeriu haver melhor qualidade de vida por ano com o IFX. Análises mais detalhadas sobre o tema são necessárias para se reproduzir estes dados.

Espera-se que com os pontos apresentados nesta discussão, o cenário da DC possa ficar semelhante ao cenário do tratamento da AR no Brasil. Os reumatologistas, atualmente, podem escolher qual droga pode ser utilizada para os seus pacientes, tendo-se as duas opções igualmente liberadas para uso contínuo (IFX e ADA). Sabe-se que há situações onde um tratamento ambulatorial, com a presença do médico durante as infusões, possa ser a preferência para determinados pacientes, com o uso do IFX. Entretanto, outros casos podem ser tratados com medicação subcutânea auto-administrada (ADA), e serem acompanhados pelos médicos em consultas com determinados intervalos de tempo. $\mathrm{O}$ bom senso e a experiência clínica determinariam qual medicação seria utilizada para cada caso.

\section{CONCLUSÕES}

Na simulação de custo-minimização entre o IFX e o ADA, houve menores custos com o IFX no tratamento contínuo por um ano, em pacientes com peso abaixo de $60 \mathrm{~kg}$. Acima deste peso, o tratamento com ADA apresentou custos mais baixos.

Em caso de perda de resposta ao IFX na semana 22 de tratamento, houve menores custos com a troca para o ADA, em pacientes acima dos $40 \mathrm{~kg}$ de peso, do que com o dobro da dosagem para o IFX. Comparando-se a troca de anti-TNF com a redução do intervalo do IFX para 6/6 semanas, os custos com o uso do ADA são menores em pacientes acima dos 60 kg. Comparando-se a perda de resposta às duas drogas, num cenário sem a troca de medicação, há menores custos ao se iniciar e resgatar o tratamento com o ADA do que com o dobro da dose do IFX em pacientes a partir dos $60 \mathrm{~kg}$ de peso.

$\mathrm{Na}$ faixa de peso médio estimada para a DC (entre 60 e $80 \mathrm{~kg}$ ), o tratamento com o ADA se mostrou menos oneroso que o IFX em todas as situações simuladas. 
ABSTRACT: Introduction: there are special concerns with costs of anti-TNF therapy in CD. The purpose of this study was a costminimization analysis with IFX and ADA in induction and maintenance of remission for one year, in different weight groups. Method: pharmacoeconomics simulation of cost-minimization in CD management. The total were based only in the drug costs in public and private health systems. Indirect findings such as infusion costs were not analyzed. Results: the cost with IFX treatment for one year, in public system, was $R \$ 29.411,12$ (between 20 and $40 \mathrm{~kg}$ ), $R \$ 44.116,68$ (between 41 and $60 \mathrm{~kg}$ ), $R \$$ $58.822,24$ (between 61 and $80 \mathrm{~kg}$ ) e $R \$ 73.527,80$ (between 81 and $100 \mathrm{~kg}$ ). The cost with ADA treatment was $R \$ 52.045,16$, not related to weight differences. Private system and loss of response simulation findings are described in the article. Conclusions: there were lower costs with IFX below $60 \mathrm{~kg}$, and with ADA above this specific weight. In loss of response to IFX situations, there were lower costs with changing to ADA strategy above $40 \mathrm{~kg}$ of weight.

Key words: Crohn's Disease; Biological Therapy; Tumor Necrosis Factor-alpha; Economics; Costs and Cost Analysis.

\section{REFERÊNCIAS}

1. Baumgart DC, Sandborn WJ. Inflammatory bowel disease: clinical aspects and established and evolving therapies. Lancet 2007;369:1641-1657.

2. Kotze LMS, Kotze PG, Parolin MB. Doença de Crohn. In: Renato Dani. (Org.). Gastroenterologia Essencial. 2nd ed. Rio de Janeiro: Guanabara Koogan; 2001. p. 276-295.

3. Travis SPL, Stange EF, Lémann M, Öresland T, Chowers Y, Forbes A, et al. European evidence based consensus on the diagnosis and management of Crohn's disease: current management. Gut 2006;55(Suppl I):i16-i35.

4. Crohn BB, Ginzburg L, Oppenheimer MD. Regional ileitis: a pathologic and clinical entity. JAMA 1932;99(6):1323-1329

5. Koltum WA. The future of surgical management of inflammatory bowel disease. Dis Colon Rectum 2008,51(6):813-817.

6. Vermeire S, Van Assche G, Rutgeerts P. Review article: altering the natural history of Crohn's disease - evidence for and against current therapies. Aliment Pharmacol Ther 2006;25:3-12.

7. Van den Brande JMH, Braat H, Van den Brink GR, Versteeg $\mathrm{HH}$, Bauer CA, Hoedemaeker I, et al. Infliximab but not Etanerecept induces apoptosis in lamina propria Tlymphocytes from patients with Crohn's disease. Gastroenterology 2003;124:1774-1785.

8. Targan SR, Hanauer SB, Van Deventer SJH, Mayer L, Present DH, Braakman T, et al. A short-term study of chimeric monoclonal antibody cA2 to tumor necrosis-factor á for Crohn's disease. N Engl J Med 1997;337(15):1029-1035.

9. Rutgeerts P, Van Assche G, Vermeire S. Review article: infliximab therapy for inflammatory bowel disease - seven years on. Aliment Pharmacol Ther 2006;23:451-463.

10. Hanauer SB, Sandborn WJ, Rutgeerts P, Fedorak RN, Lukas $\mathrm{N}$, Macintosh D, et al. Human anti-tumor necrosis factor monoclonal antibody (adalimumab) in Crohn's disease: the CLASSIC-I trial. Gastroenterology 2006;130(2):323-333.

11. Colombel JF. Efficacy and safety of adalimumab for the treatment of Crohn's disease in adults. Expert Rev Gastroenterol Hepatol 2008;2(2):163-176.
12. Hanauer SB, Feagan BG, Lichtenstein GR, Mayer LF, Schreiber S, Colombel JF, et al. Maintenance infliximab for Crohn's disease: the ACCENT I randomised trial. Lancet 2002;359:1541-1549.

13. Sands BE, Anderson FH, Bernstein CN, Chey WY, Feagan BG, Fedorak RN, et al. Infliximab maintenance therapy for fistulizing Crohn's Disease. N Engl J Med 2004;350:876885 .

14. Colombel JF, Sandborn WJ, Rutgeerts P, Enns R, Hanauer $\mathrm{SB}$, Panaccione R, et al. Adalimumab for maintenance of clinical response and remission in patients with Crohn's disease: the CHARM trial. Gastroenterology 2007;132(10):52-65.

15. Sandborn WJ, Rutgeerts P, Enns R, Hanauer SB, Colombel $\mathrm{JF}$, Panaccione R, et al. Adalimumab induction therapy for Crohn's disease previously treated with infliximab: a randomized trial. Ann Intern Med 2007;146:829-838.

16. Rutgeerts P, Feagan BG, Lichtenstein GR, Mayer LF, Schreiber $\mathrm{S}$, Colombel JF, et al. Comparison of scheduled and episodic treatment strategies of infliximab in Crohn's disease. Gastroenterology 2004;126:402-413.

17. Feagan BG, Panaccione R, Sandborn WJ, D'Haens GR, Schreiber S, et al. Effects of adalimumab therapy on incidence of hospitalization and surgery in Crohn's disease: results from the CHARM study. Gastroenterology 2008;135(5):1493-9

18. Scheinberg M, Goldenberg J, Souza BD. Análise Custominimização da Terapia Anti-TNF no Brasil. Rev Bras Med 2005;62(3):90-95.

19. Sandborn WJ, Hanauer SB, Rutgeerts P, Fedorak RN, Lukas $\mathrm{M}$, Macintosh D, et al. Adalimumab for maintenance treatment of Crohn's disease: results of the CLASSIC II trial. Gut 2007; 56:1232-1239.

20. Sandborn WJ, Colombel JF, Enns R, Feagan BG, Hanauer SB, Lawrence IC et al. Natalizumab induction and maintenance therapy for Crohn's disease. N Engl J Med. 2005;353(18):19121925.

21. Revista Kairos 2009; 243:156,192.

22. CAP (Coeficiente de adequação do preço): Comunicado no 15 de 28 de dezembro de 2007 - Publicado no Diário Oficial da União de 31.12.2007. 
23. Regueiro M, Siemanowski B, Kip KE, Plevy S. Infliximab dose intensification in Crohn's disease. Inflamm Bowel Dis 2007;13(9):1093-1099.

24. Wyneski MJ, Green A, Kay M, Wyllie R, Mahajan L. Safety and efficacy of adalimumab in pediatric patients with Crohn's disease. J Pediatr Gastroenterol Nutr 2008;47(1):19-25.

25. Oldemburg B, Hommes D. Biological therapies in inflammatory bowel disease: top-down or bottom-up? Curr Opin Gastroenterol 2007;23(4):395-9.

26. Van Assche G, Vermeire S, Rutgeerts P. Management of loss of response to anti-TNF drugs: change the dose or change the drug? JCC 2008;2:348-351.

27. Wu EW, Mulani PM, Yu AP, Tang J, Pollack PF. Loss of treatment response to infliximab maintenance therapy in
Crohn's disease: a payor perspective. Value Health 2008;5(2):820-829

28. Kaplan GG, Hur C, Korzenik J, Sands BE. Infliximab dose escalation vs. initiation of adalimumab for loss of response in Crohn's disease: a cost-effectiveness analysis. Aliment Pharmacol Ther 2007;26:1509-20.

\section{Endereço para correspondência:}

DR. PAULO GUSTAVO KOTZE

Rua Jaime Balão, 975 - ap. 106 - Curitiba - PR

CEP 80040-340

E-mail:pgkotze@hotmail.com

FAX: 4132537838 